\title{
Medio y contexto de la trabajadora social de atención primaria en Aragón
}

Antonio Matías Solanilla

Universidad de Zaragoza

amatias@unizar.es

\section{Antonio Eito Mateo}

Universidad de Zaragoza

antoni@unizar.es

\section{Chaime Marcuello Servós}

Universidad de Zaragoza

chaime@unizar.es
Este artículo presenta los resultados preliminares de una investigación en curso sobre el papel de las trabajadoras sociales - pues son mayoritariamente mujeres- en la ejecución y puesta en práctica de las políticas públicas en materia de servicios sociales en Aragón. A partir de un análisis del imaginario de las profesionales, recogido mediante entrevistas en profundidad, se revisan las experiencias, posiciones y valoraciones de su labor en los servicios sociales de atención primaria aragoneses. Desde sus discursos se revisan procesos, trayectorias y situación actual del sistema de protección, así como la profesión y las expectativas respecto a ella. Las transformaciones sociales, políticas y económicas vividas en nuestro país han marcado y configurado la labor diaria en los centros donde se gestionan estos servicios sociales. Su éxito depende del buen hacer de los diferentes profesionales, donde destaca como figura esencial la trabajadora social. Es la referencia de usuarios y responsables políticos.

\section{Palabras clave}

Servicios sociales de atención primaria, política social, trabajadora social, administración local.

Oinarrizko gizarte zerbitzuak, gizarte politika, gizarte langilea, tokiko administrazioa. 


\section{Introducción}

A finales de la década de los setenta, con el inicio de la Transición en la sociedad española se dieron los primeros pasos para crear un sistema de servicios sociales. Sistema que en las décadas posteriores se ha consolidado en la sociedad española siendo un pilar básico de nuestro "frágil” modelo de Estado de Bienestar.

La teoría (Casado, 1987) y las diferentes legislaciones autonómicas ${ }^{1}$ dividen tradicionalmente a los servicios sociales en servicios sociales generales, también conocidos como de atención primaria y servicios sociales especializados.

Los servicios sociales de atención primaria han tenido su particular desarrollo en el Estado español. En la gestión y aproximación técnica a ellos se han posicionado mayoritariamente las trabajadoras sociales, quienes han sido las verdaderas impulsoras de este proceso de transformación que ha vertebrado España en materia de política social.

No en vanguardia, pero sí en posiciones cercanas, hay que destacar el desarrollo de los servicios sociales de atención primaria en Aragón. En 1980 se crearon los primeros centros de servicios sociales en Zaragoza y en 1983 el primer servicio social de base rural.

Casi cuarenta años después de la creación de los primeros servicios sociales municipales aragoneses, es pertinente revisar un poco la historia de su creación y analizar ese proceso a partir de sus profesionales más representativos, mostrando las claves de la situación actual y anticipando algunos elementos de futuro.

\section{Una aproximación terminológica y legislativa}

Los servicios sociales de atención primaria se pueden definir, tomando como referencia el artículo 13 de la Ley 5/2009 de 30 de junio de Servicios Sociales de Aragón ${ }^{2}$, como "servicios públicos de carácter polivalente y comunitario cuya organización y personal están al servicio de toda la población". Constituyen el primer nivel del sistema público de servicios sociales y proporcionan una atención que tiene por objeto favorecer el acceso a los recursos de los sistemas de bienestar social, generando alternativas a las carencias o limitaciones en la convivencia, favoreciendo la inclusión social y promoviendo la cooperación y solidaridad social en un determinado territorio.
Legislativamente hablando, hay que resaltar que en la Constitución Española de 1978 no se encuentra ninguna referencia al término "servicios sociales" y mucho menos a la denominación "servicios sociales generales”, “de base”, “de atención primaria”, etc. La Constitución de 1978, en el Título I, “De los Derechos y deberes fundamentales", establece los derechos de la persona (art. 10), igualdad ante la ley (art. 14), educación (art. 27), protección a la familia y la infancia (art. 39), seguridad social (art. 41), protección a la salud (art. 43), participación de la juventud (art. 48), atención a disminuidos (art. 49), tercera edad (art. 50), etc., pero en ningún momento se hace alusión a conceptos específicos de "asistencia social” o "servicios sociales". Hasta el artículo 148 , apartado 1.20, no se habla de las competencias de las comunidades autónomas en asistencia social. Luego, en el artículo 149, 1.17, se enumeran las competencias exclusivas del Estado y se cita "Legislación básica y régimen económico de la Seguridad Social”.

Aunque la Ley $6 / 1982$ de 20 de mayo de Servicios Sociales de Euskadi ya deja intuir el papel fundamental de la Administración Local en la implementación de los servicios sociales de atención primaria, no hay una referencia expresa a tal efecto. Habrá que esperar a la Ley 11/1984 de 6 de Junio de Servicios Sociales de la Comunidad Autónoma de Madrid para encontrar una referencia expresa a la división de servicios sociales generales y especializados atribuyendo al municipio la competencia y titularidad sobre ellos.

Posteriormente, la Ley 7/1985 de Bases de Régimen Local refrenda esa competencia y titularidad al estipular en sus artículos 25, 2.k y 26, 1.c ${ }^{3}$ que la prestación de servicios sociales y de reinserción social es de competencia municipal y obligatoria para los municipios mayores de $\mathbf{2 0 . 0 0 0}$ habitantes, aunque no concreta en qué consisten esos servicios.

\section{Un poco de historia}

Aunque, tal y como reconoce García (1988), hay reseñas no suficientemente documentadas de la existencia de primigenias experiencias de servicios sociales comunitarios públicos en los años sesenta en pueblos llamados "de colonización", tenemos que cifrar los primeros pasos ideológicos de un intento de crear una red de servicios sociales de atención primaria públicos en la época preconstitucional de finales de los años setenta del pasado siglo XX. Concretamente, es en el año 1977 cuando en las III Jornadas Nacionales de Asistentes Sociales organizadas por la Federación Española de Asociaciones de Asistentes Sociales (FEDAS), más conocidas como "las Jornadas de Pamplona", cuando

${ }^{3}$ Aunque somos sabedores de que la Ley $27 / 2013$ de 27 de diciembre de racionalización y sostenibilidad de la Administración Local modificó los citados artículos, hacemos referencia a la redacción original, dado que estamos analizando la implantación de los servicios sociales de atención primaria. 
se habla abiertamente de la necesidad de agencias locales de bienestar social: "Se hace necesario en cada distrito, barrio y pueblos, la existencia de una agencia de bienestar social, formada por un equipo interprofesional, vinculada directamente al ayuntamiento o diputación (según el ámbito territorial que abarque), que tendrá como misión la puesta en marcha de todos los Servicios Sociales necesarios en la zona, así como el asesoramiento técnico de la comunidad vecinal” (Fedas, 1977: 45).

Fruto de esas jornadas y del trabajo de reflexión realizado en esa época aparece en 1979 el libro Introducción al Bienestar Social, publicado por Patrocinio De Las Heras y Elvira Cortajarena. Tal y como recogen en el prólogo a la reedición de 2014: "El texto contiene el análisis histórico de la Acción Social, la evolución de sus Formas Históricas y las propuestas para el Bienestar Social [...]. Incorpora también un modelo municipalista de intervención profesional a través de la realidad local en dicho contexto histórico" (De las Heras y Cortajarena, 2014: 12).

En este sentido, hay que mencionar dos experiencias dentro del Estado español. Por un lado, la creación en el Ayuntamiento de Vitoria-Gasteiz de las "oficinas municipales" en el año 1976. Estas se definen como instrumento idóneo - bidireccional- para llevar al Ayuntamiento la realidad y opinión de los barrios, y para llevar a los barrios la planificación y las directrices generales del ayuntamiento. Estas oficinas fueron un elemento de planificación e integración al mismo tiempo que se consideraron como equipamiento social de los barrios y también como cauces de participación e información. Al frente de cada una de ellas estaba una asistente social (CGTS, 1988).

Por otro lado, en Cataluña se fraguó paralelamente lo que se ha denominado Escuela Catalana de Servicios Sociales (Casado, 1987), compuesta por una serie de destacadas profesionales del trabajo social que habían formado el GITS (Grup d’Investigació i Formació en Treball Social) vinculadas al Partido Socialista de Cataluña y que, con la victoria en las elecciones de 1979, que aúpan al PSC-PSOE al Ayuntamiento de Barcelona, consiguieron crear en dicho ayuntamiento un área de servicios sociales, la primera experiencia en España en este aspecto.

A la estela de la experiencia de los ayuntamientos de Vitoria-Gasteiz y de Barcelona, otros ayuntamientos de grandes ciudades iniciaron en la década de los ochenta la creación de sus propias áreas de servicios sociales o bienestar social, como fue el caso de Zaragoza.

Las III Jornadas de Servicios Sociales Municipales celebradas los días 1, 2 y 3 de julio de 1983 en Castelldefels (Barcelona), organizadas por el PSCPSOE supusieron la asunción por parte del PSOE de los postulados en servicios sociales ideados por los movimientos antes descritos y que poco a poco se fueron implantando en el conjunto del Estado.
En dichas jornadas se trazó la primera hoja de ruta de la implantación de un sistema de servicios sociales en todo el territorio, empezando por una instancia municipal. "En una etapa inicial hay que empezar por un programa fuerte de servicios sociales comunitarios, de los cuales se tendrán que derivar los servicios sociales especializados, si en realidad responden a una planificación resultante del estudio y trabajo de base" (PSOE-Secretaría Federal de Acción Social, 1984: 47).

La victoria del partido socialista en la mayoría de comunidades autónomas y municipios en las elecciones autonómicas y locales de mayo de 1983 , unida a la obtenida por mayoría absoluta en las generales de octubre de 1982, hicieron que aquella "hoja de ruta" se fuese instaurando progresivamente en todo el territorio.

En 1988 se firmó el Plan Concertado de Prestaciones Básicas de Servicios Sociales a iniciativa del Ministerio de Trabajo y Seguridad Social con el objeto de articular la cooperación económica entre Administraciones y con la finalidad de lograr la colaboración entre la Administración del Estado y las Comunidades Autónomas para financiar conjuntamente la red de atención de servicios sociales. Esto sirvió, por un lado, para garantizar su supervivencia económica y, por otro, para homogenizar mínimamente los servicios en todo el territorio español.

\subsection{El caso aragonés}

La historia de los servicios sociales de atención primaria en el territorio aragonés difiere levemente del resto de España. Por lo que se refiere al Ayuntamiento de Zaragoza, se inicia la andadura en materia de servicios sociales en el año 1980, siendo alcalde Sáinz de Varanda. Para ello se adaptan de manera propia las experiencias de Vitoria y Barcelona, todo ello bajo el mandato de la concejala de Servicios Sociales María Arrondo. En esta primera legislatura democrática se crearon ocho centros de servicios sociales municipales en barrios de la ciudad (Gutiérrez, 1999).

No obstante, la diferencia está en el medio rural, que en un primer momento y desde el Gobierno preautonómico de Aragón se considera al resto del territorio aragonés, con la excepción de la capital, Zaragoza. La primera referencia a señalar es el Boletín Oficial de Aragón de 24 de marzo de 1983, dos meses antes de las elecciones autonómicas y locales y cuatro meses antes de la celebración de las Jornadas de Castelldefels, cuando se recoge la Orden de 9 de marzo del mismo año por la que se establecían las normas generales reguladoras de la Convocatoria Regional Unificada de Atención Social. En dicha orden se establece la primera convocatoria de subvenciones para "ayudas para creación, equipamiento y mantenimiento de servicios 
sociales de base" ${ }^{4}$. Una lectura pormenorizada de dicha orden arroja varios elementos dignos de destacar:

- Las actividades de los servicios sociales de base tienen por finalidad la atención de los problemas de la comunidad residente en la zona, su desarrollo y la mejora de su calidad de vida.

- Se determina que estas ayudas son preferentemente para las corporaciones de más de 3.000 y menos de 50.000 habitantes $^{5}$. Se opten también a estas ayudas si se encuentran en los límites poblacionales fijados.

- Se posibilita también que puedan solicitar estas ayudas "las asociaciones o personas jurídicas que hayan iniciado en una zona experiencias de desarrollo comunitario siempre que coordinen su actividad con los ayuntamientos correspondientes".

De esa primera convocatoria nacen los primeros servicios sociales de base de Aragón, concretamente la mancomunidad de municipios de Flumen Monegros (zona sur de la provincia de Huesca y algunos municipios de la de Zaragoza con relaciones y vinculaciones históricas, embrión de la posterior comarca de Los Monegros), y de los ayuntamientos de Fraga, Andorra y Huesca. Los municipios afectados eran veinte y el número de habitantes 83.117.

Tras las elecciones autonómicas de 1983 y con la victoria socialista se constituye el primer gobierno autonómico, que ejerce una apuesta decidida por los recién creados servicios sociales de base. Antes de que acabe ese mismo año se dicta el Decreto $114 / 1983$ de 29 de diciembre (BOA de 16 de enero de 1984) en el que se publica la primera convocatoria exclusiva de subvenciones para creación y mantenimiento de los servicios sociales de base para el año 1984. Respecto a la anterior subvención hay dos modificaciones de importancia: desaparece la posibilidad de que asociaciones y personas jurídicas puedan acceder a este tipo de subvenciones, con lo que ya se confiere una responsabilidad exclusivamente pública sobre los servicios sociales de base y, por otra, se modifican los límites poblacionales; la población mínima municipal para solicitar la subvención tiene que ser de diez mil habitantes y si son agrupaciones de municipios tiene que ser de cinco mil habitantes.

Con el Decreto 114/83 de 29 de diciembre, el Gobierno aragonés hace una apuesta firme y decidida por constituir una red de servicios sociales de atención primaria en todo el territorio. Desde el recién constituido Gobierno y con la

${ }^{4}$ Nomenclatura que se adopta en 1983 para designar a los servicios sociales de atención primaria en el medio rural aragonés.

5 Solo la ciudad de Zaragoza superaba en aquella fecha los 50.000 habitantes. posibilita que agrupaciones de varios municipios

el asesoramiento y diseño de esta política social, se inicia una expansión y "venta" de la idea de servicios sociales de base entre los responsables políticos de municipios y mancomunidades para la creación de estos servicios (Báñez, 2005).

Esta "venta" fue efectiva, ya que si a principios del año 1984 eran cuatro los servicios sociales que estaban en marcha, su asentamiento y multiplicación en el territorio aragonés fue fulgurante, tal y como se recoge en la Tabla 1.

Tabla 1. Evolución de los servicios sociales de base en Aragón (1983-1988)

\begin{tabular}{|l|c|c|c|c|}
\hline Año & $\begin{array}{c}\text { Número de } \\
\text { servicios } \\
\text { sociales de } \\
\text { base }\end{array}$ & $\begin{array}{c}\text { Asistentes } \\
\text { sociales }\end{array}$ & Municipios & $\begin{array}{c}\text { Población } \\
\text { total }\end{array}$ \\
\hline 1983 & 4 & 6 & 20 & 83.117 \\
\hline 1984 & 23 & 31 & 210 & 279.455 \\
\hline 1985 & 29 & 42 & 286 & 341.679 \\
\hline 1986 & 34 & 52 & 369 & 403.780 \\
\hline 1987 & 38 & 59 & 395 & 426.673 \\
\hline 1988 & 48 & 74 & 480 & 535.765 \\
\hline
\end{tabular}

Fuente: Elaboración propia a partir de guías de los servicios sociales básicos publicadas por la Consejería de Sanidad, Bienestar Social y Trabajo del Gobierno de Aragón (no incluye los datos de la ciudad de Zaragoza).

Estos datos indican que, solo cinco años después del inicio de los servicios sociales de base, estos ya amparaban, una vez restada la población de Zaragoza capital, a aproximadamente al $87,5 \%$ de la población aragonesa y al $66 \%$ de los municipios. Por tanto, no es de extrañar que las primeras jornadas españolas sobre servicios sociales en el medio rural se celebrasen en Aragón, concretamente en Graus y Daroca en 1985.

Al finalizar 1993, los servicios sociales de base alcanzaron una cobertura territorial del $91,6 \%$ del territorio aragonés y un porcentaje del 98,9\% de la población (Gutiérrez, 1999).

En la Tabla 2 se puede observar cómo se desarrolló la implantación y consolidación de los servicios sociales de base en el territorio en la década de los noventa atendiendo a tres catas en los años 1994, 1996 y 2000 . En esa época, la Consejería de Sanidad, Bienestar Social y Trabajo publicó directorios internos que muestran esta información.

Tabla 2. Desarrollo de los servicios sociales de base en la década de los noventa

\begin{tabular}{|l|c|c|c|c|}
\hline Año & $\begin{array}{c}\text { Número de } \\
\text { servicios } \\
\text { sociales de base }\end{array}$ & $\begin{array}{c}\text { Población } \\
\text { total }\end{array}$ & $\begin{array}{c}\text { Municipios } \\
\text { atendidos }\end{array}$ & $\begin{array}{c}\text { Municipios } \\
\text { sin } \\
\text { atención }\end{array}$ \\
\hline 1994 & 69 & 583.113 & 670 & 60 \\
\hline 1996 & 69 & 585.231 & 689 & 41 \\
\hline 2000 & 70 & 585.872 & 710 & 20 \\
\hline
\end{tabular}

Fuente: Elaboración propia a partir de guías de los servicios sociales básicos publicadas por la Consejería de Sanidad, Bienestar Social y Trabajo del Gobierno de Aragón (no incluye los datos de la ciudad de Zaragoza). 
En el año 1988, los datos mostraban 48 servicios sociales de base y una población atendida que ascendía a 535.765 ciudadanos. Seis años después, la creación de servicios sociales de base había aumentado en un $43,75 \%$, mientras que la población en el mismo periodo de tiempo había aumentado solamente un $8,8 \%$ y la variación en datos hasta el año 2000 es prácticamente inapreciable, pese a que el número de poblaciones no atendidas por un servicio iba menguando considerablemente. Esta situación muestra que la década de los noventa se caracterizó por la creación de algunos nuevos servicios sociales de base, pero también por la escisión en otros más pequeños con el objeto de ser más operativos, alentada también por los responsables de los ayuntamientos, que vieron los servicios sociales de base y la labor de sus profesionales como oportunidad, en tanto que unos servicios que benefician a toda la población.

En 1999, el consejero de Sanidad, Bienestar Social y Trabajo, en una publicación de la mancomunidad del Alto Gállego afirmaba que “la atención primaria en servicios sociales ofrece sus prestaciones a los ciudadanos a través de la red pública de las corporaciones locales. Aragón, por cierto es una de las pocas comunidades autónomas que ha conseguido por este sistema una cobertura de población del $100 \%$ ".

Legislativamente hay que destacar tres apuntes más para entender la situación de los servicios sociales de atención primaria en Aragón:

- Ley 4/1987, de 25 de marzo, de Ordenación de la Acción Social en Aragón (LOAS). En el articulado de esta ley se configura una primera ordenación legislativa del sistema de servicios sociales, donde se especifica que podrán tener carácter comunitario o dirigirse a un sector específico de la sociedad. En esta ley de primera generación, los servicios sociales comunitarios se denominan "servicios sociales de base" y "configuran la estructura básica de la acción social, correspondiendo su gestión a los ayuntamientos".

- Ley 7/99, de 9 de abril, de Administración Local de Aragón. En su artículo 44 a) indica que dentro de los servicios municipales obligatorios se encuentra la obligación de "gestión de los servicios sociales de base" pudiendo gestionarlos por sí solos o asociados a otras entidades locales.

- El proceso comarcalizador de Aragón con el Decreto Legislativo 1/2006, de 27 de diciembre, que refunde toda la legislación que lo reguló en el último decenio del siglo XX. En su artículo 9.1.6., se indica como competencia propia de la comarca la acción social.

\section{El papel de las trabajadoras sociales}

Analizar y relatar, aunque sea mínimamente, cómo fue el nacimiento y cuál ha sido la trayectoria de los servicios sociales de atención primaria obliga a hacer constantes referencias a uno de sus actores principales, la trabajadora social. Tal y como indica Gil Parejo (2010), a partir de la Constitución Española y la llegada de la democracia, el trabajo social define su espacio profesional en los servicios sociales, llegando a veces incluso a identificarlo como propio. En buena medida puede ser porque el PSOE, como partido político que en el final de los setenta y principio de los ochenta lideró la creación del sistema de servicios sociales, contaba entre sus filas con asistentes sociales que se volcaron en un arduo trabajo del que luego se ha beneficiado toda la profesión.

En las jornadas de Pamplona, en la ponencia "Planificación, organización y desarrollo de los servicios sociales y el bienestar social”, elaborada por el comité organizador ${ }^{6}$, ya se indica que "las agencias de bienestar social estarán a cargo de asistentes sociales que asumirán las funciones de coordinación y promoción de la acción social" (Fedaas 1977: 47). Idea refrendada posteriormente por De las Heras y Cortajarena en su libro Introducción al Bienestar Social, donde a la hora de hablar de las unidades de bienestar social afirman: "La intervención técnica se realiza específicamente a través de asistentes sociales a quienes compete, en todo programa de acción directa con la comunidad, la atención global a las necesidades sociales en relación a las prestaciones y servicios que precisan [...] el asistente social de la unidad (asistente social polivalente) articula el trabajo social en la comunidad con el trabajo social por área de necesidad (sanidad, educación, empresa, instituciones, etc.), de la misma forma que el médico de cabecera vincula la atención médica general con los servicios de especialidad" (De las Heras y Cortajarena, 1978: 143).

Por su parte, la Escuela Catalana de Servicios Sociales apoyó también estas tesis como autoras ${ }^{7}$ de la ponencia "Los Centros Municipales de Servicios Sociales" dentro de las III Jornadas de Servicios Sociales organizadas por el PSOE, recogidas por el volumen 14 de Cuadernos de Acción Social. "Los asistentes sociales polivalentes son el equipo básico del centro por el carácter generalista y global de su trabajo que abarca la totalidad de las competencias en sus tres dimensiones individual y familiar, institucional y comunitaria” (PSOE, 1984: 85).

García (1987), dando por hecho también el protagonismo de los asistentes sociales y fruto de las jornadas de Daroca, reflexiona sobre el perfil profesional que en el inicio de la profesión debían

${ }^{6}$ El comité organizador de dichas Jornadas estaba presidido por María Patrocinio Las Heras Pinilla, autora posterior conjuntamente con Elvira Cortajarena de Introducción al Bienestar Social. Patrocinio de las Heras fue coordinadora de Políticas de Bienestar Social del PSOE entre 1981 y 1983 . Posteriormente fue directora general de Acción Social del Imserso, del Inas y del Fonas, entre 1983 y 1990.

7 El texto viene firmado en orden alfabético por Montserrat Colomer, Rosa Domenech, Montserrat Flaquer, María Francesca Masgoret, María Teresa Massons y Gloria Rubiol, todas ellas miembros del GITS. 
tener específicamente los asistentes o trabajadores sociales de servicios sociales de atención primaria rurales. Merece la pena releer esas características que, por otra parte, no se enseñaban en las antiguas escuelas y rara vez en facultades actuales:

- Imaginación, ya que en muchas ocasiones había que dar respuestas novedosas a situaciones peculiares sin modelos previos de referencia.

- Capacidad de síntesis, dada la formación extensiva y la multitud de temas que se abordaban.

- Capacidad de empatizar.

- Aptitudes de dinamización para generar dinamismos que entonces se consideraban "encasillados en la pasividad".

- Capacidad de unir, coordinar y multiplicar recursos para conseguir los objetivos (evidentemente, relacionado con la capacidad de imaginación).

- Paciencia. No hay que olvidar en ningún momento que se estaba viviendo una experiencia única de puesta en marcha de una nueva política pública, de creación de un sistema de servicios que, salvo alguna experiencia aislada, era totalmente novedoso y desconocido para los usuarios, para los políticos y para los profesionales, por lo que los resultados podían demostrarse a largo plazo y los errores y sinsabores podían estar a la orden del día.

La creación de un nuevo sistema de protección fácilmente debió de sembrar de dudas a políticos locales y autonómicos. Si se bucea un poquito en los archivos de las Cortes de Aragón de esa primera legislatura (1983-1987), aparecen diversas referencias tanto a la creación de los servicios sociales de base como a los asistentes sociales, y posteriormente trabajadores sociales, con un protagonismo que difícilmente han podido llegar a tener en ese escenario en otro momento. Con poco más de un año de vida de dicha legislatura, el consejero Arola Blanquet afirmaba en una comisión de Trabajo, Sanidad, Seguridad Social y Servicios Sociales ${ }^{8}$ que "como objetivo básico de mi departamento y de la acción queríamos desarrollar en el marco de los servicios sociales, básicamente la creación de un sistema", estableciendo como uno de los elementos básicos de ese sistema "la creación en nuestra comunidad autónoma de los servicios comunitarios que eran la puerta de entrada al sistema". En la misma comparecencia hablaba de las bondades de los nuevos servicios comunitarios, que todavía no tenían dos años de vida: "hemos renunciado a continuar con una tradición singular de parcheadores 0 apagafuegos, o de beneficencia, en beneficio de un sistema de base. Y si logramos esto, nosotros o quien haya de sustituirnos podrá avanzar en la configuración de todo tipo de servicios especializados".

${ }^{8}$ Comisión celebrada el martes 9 de abril de 1985 y recogida en el Diario de Sesiones de las Cortes de Aragón, número 2, de la Legislatura I.
En el transcurso de dicha comparecencia, el consejero afirma "que ha mejorado la calidad de vida en las zonas en que se han implantado los servicios sociales de base". En la misma comisión y en preguntas e interpelaciones diferentes a lo largo de la legislatura se iban ofreciendo datos del desarrollo de estos servicios y de sus profesionales, llegando por ejemplo a discutirse en 1985 si la labor de los asistentes sociales de los servicios sociales de base estaba o no demasiado burocratizada9. En dicha interpelación el propio consejero se llegaba a plantear: “¿Estoy satisfecho de cómo están trabajando los asistentes sociales? Globalmente, evidentemente la respuesta es afirmativa. Los han definido, por ejemplo, como gentes que están, como retoños que están creando brotes de participación, y no es una definición. Estoy globalmente satisfecho".

Tal fue el protagonismo en el inicio de la década de los ochenta en la Comunidad Autónoma Aragonesa de los servicios sociales de base, que en el debate de totalidad del Proyecto de Ley de Ordenación de Acción Social el entonces diputado Biel afirmó que era "una ley donde han metido los servicios sociales de base, que parece que es su única obsesión a lo largo de toda la legislatura" ${ }^{10}$.

Realmente, obsesión o no, el caso es que en la primera legislatura del Gobierno autonómico aragonés se apostó decididamente por la constitución de servicios sociales de base y, por lo tanto, por la figura de los asistentes sociales, que fueron los y las artífices y el principal recurso (Báñez, 2005).

Y aunque, efectivamente, con el inicio de los centros municipales de servicios sociales, o en su denominación rural los servicios sociales de base, se inicia la contratación sistemática de trabajadores sociales que se ponen al frente de ellos, legislativamente hablando no encontramos en el inicio ninguna norma que obligue esta contratación. En el caso de Aragón, la orden de 9 de marzo de 1983 y el Decreto 114/83 no mencionan en ningún momento a la figura del asistente social o trabajador social.

Hay que esperar a la Ley 5/2009 de Servicios Sociales de Aragón para encontrar una referencia concreta a los "profesionales del trabajo social" cuando cita en su artículo 14.3: "Dentro del equipo multidisciplinar se integran los Servicios Sociales de Base, como unidades de trabajo social que prestan su atención directa en todos los municipios de su ámbito respectivo, facilitando tanto el acceso al sistema de servicios sociales como al profesional de referencia de dicho ámbito".

La aprobación del Decreto 184/2016 (BOA, $28 / 12 / 2016)$, por el que se regula la organización y

9 Interpelación 10/85 formulada por el diputado Agudo González con relación a los criterios de la Diputación General de Aragón sobre la elaboración de servicios sociales recogida en el Diario de Sesiones de las Cortes de Aragón, número 32, sesión plenaria 09/05/1985.

${ }^{10}$ Diario de Sesiones de las Cortes de Aragón, número 63. 
funcionamiento de los centros de servicios sociales, designa concretamente al trabajador social como profesional de referencia y regula el número mínimo de trabajadores sociales que tiene que haber por centro de servicios sociales en función del territorio y la población que abarcan.

\section{Un análisis de los discursos}

Se puede afirmar que actualmente la ciudadanía aragonesa, al igual que tiene un médico de familia del servicio público de salud asignado, tiene también una trabajadora social asignada como profesional de referencia que presta sus servicios en un centro de servicios sociales bien sea municipal o comarcal. Estas profesionales son, con mucha diferencia, la categoría profesional más numerosa de dichos centros de servicios sociales, que constituyen la puerta de entrada al sistema de servicios sociales y que es uno de los cuatro pilares de nuestro modelo de bienestar. Sin embargo, salvo honrosas excepciones como Marco (1988) y Báñez (2005), es difícil encontrar referencias bibliográficas sobre los inicios de la profesión o la situación actual de las profesionales que hagan referencia a algo más que no sea la presentación de experiencias propias laborales a través de la exposición de proyectos o desarrollo de programas y prestaciones concretas que, siendo muy interesantes, no encajan en esta investigación. Aquí se aborda ese espacio vacío entrevistando a trabajadoras sociales de centros de servicios sociales comarcales, buscando su reflexión sobre sus inicios en la profesión, cómo ha sido el desarrollo y cuál es su percepción sobre el futuro tanto de la profesión como de los servicios sociales de atención primaria.

Se ha obviado deliberadamente en esta primera aproximación a las profesionales de los servicios sociales de atención primaria "urbanos". En las comarcas, la distancia con la capital y su dirección provincial, así como la falta de recursos específicos, sigue haciendo a esta profesional una trabajadora social polivalente con una realidad diferenciada del medio urbano.

Se han seleccionado, por tanto, un total de 14 profesionales en un rango de experiencia laboral entre los 8 y los 32 años, a las que se ha entrevistado entre los meses de noviembre y diciembre de 2016 , tal y como recoge la Tabla 3.

La asistente social de mediados de los ochenta se caracterizaba por la itinerancia, juventud, soledad e inexperiencia, en medio de un espacio y unas circunstancias para las que los manuales de las escuelas de entonces servían de poco (García, 1990). En todas las entrevistas salen a relucir algunos lugares comunes compartidos por las profesionales. Aquellas que comenzaron en su territorio un servicio social de base, bien sea a mediados de los ochenta (E1, E12) como en otras oleadas a principios de los años noventa (E8, E10, E11), hablan de mucha ilusión, ganas y muchas expectativas, pero a la vez de una sensación de soledad. Jornadas de trabajo maratonianas, con horarios más que flexibles, trabajando mañanas, tardes y noches, tal y como indican algunas de estas pioneras: "Empezar, llegar aquí y no haber nada. Nada. Los ayuntamientos se apuntaban porque daban dinero, pero no había nada. Había que pedir en los ayuntamientos si te dejaban la máquina de escribir” (E1).

Y el trabajo siempre fue el mismo. Empezar apostando por la animación comunitaria, promoviendo el asociacionismo en la zona. Asociaciones de mayores, de mujeres, de consumidores, etc. ${ }^{11}$, como medio para darse a conocer y también, como reconocen, porque en los inicios las prestaciones eran

Tabla 3. Características de las profesionales entrevistadas

\begin{tabular}{|l|c|c|c|c|}
\hline Trabajadora social & Sexo & Años de experiencia & Funciones & Población comarcal \\
\hline E1 & M & 31 & Trabajadora social de estructura & 8.000 \\
\hline E2 & M & 26 & Trabajadora social de refuerzo & 8.000 \\
\hline E3 & M & 8 & Directora de centro de servicios & 8.000 \\
\hline E4 & M & 9 & Trabajador social de estructura & 8.000 \\
\hline E5 & H & 16 & Trabajadora social de estructura & 14.000 \\
\hline E6 & M & 25 & Trabajadora social de estructura & 29.000 \\
\hline E7 & M & 25 & Trabajadora social de estructura & 29.000 \\
\hline E8 & M & 26 & Trabajador social de refuerzo & 29.000 \\
\hline E9 & H & 9 & Trabajadora social de estructura & 3.000 \\
\hline E10 & M & 26 & Trabajadora social de estructura & 9.000 \\
\hline E11 & M & 26 & Trabajadora social de estructura & 9.000 \\
\hline E12 & M & 32 & Trabajadora social de estructura & 24.000 \\
\hline E13 & M & 17 & Trabajador social de estructura & 24.000 \\
\hline E14 & H & 20 & & 11.000 \\
\hline
\end{tabular}

Fuente: Elaboración propia.

${ }^{11}$ Cabe destacar que la primera publicación del Gobierno de Aragón que recoge datos del trabajo de los servicios sociales de base, Los Servicios Sociales en Aragón-1984, señala como tareas básicas de estos servicios la información, el fomento del asociacionismo y posibilitar cauces de participación. 
prácticamente inexistentes: “Al principio era abrirte, venderte, darte a conocer y eso lo conseguimos con la animación comunitaria, también porque no había más recursos" (E12).

Con la firma del Plan Concertado (1988) y con la aparición de diferentes prestaciones como las pensiones no contributivas, el Ingreso Aragonés de Inserción y la Ayuda de Integración Familiar, así como las más recientes derivadas de la Ley 39/2006 de Promoción de la Autonomía Personal y Atención a las Personas en Situación de Dependencia, más conocida como Ley de la Dependencia, todas coinciden en que el trabajo social en los servicios sociales de atención primaria se ha vuelto mucho más "prestacionista" dejando de lado la animación comunitaria. Trabajo - prestacionista- que acarrea mucha más burocracia y que representa una queja y, en el fondo, autocrítica: "Se introdujo la tecnología, bases de datos... Eso iba a sustituir al papel. No lo ha sustituido. Ha sumado. Ahí no hemos ganado tiempo y lo hemos perdido en animación comunitaria. Esa ha sido la mayor pérdida" (E7). "Se emplea mucho tiempo en trabajo administrativo y en prestaciones que no necesitan valoración y que hacemos los trabajadores sociales. A veces nos dedicamos a rellenar papeles" (E5).

Sin embargo, también hay profesionales que reivindican y recuerdan la necesidad a veces de ese papel burocrático: "Hay que rellenar solicitudes en la medida de las capacidades del usuario. Si no medimos esa capacidad, dejamos de utilizar nuestra profesión para ser puerta de entrada. A veces traducir y adaptar el lenguaje administrativo y adaptar procesos que a nuestros usuarios les pueden parecer complejos es nuestra función" (E10).

Por otra parte, el papel de los servicios sociales de atención primaria en la actualidad también genera consensos. La mayoría de las profesionales los consideran como "esenciales para el buen funcionamiento de la sociedad" (E1, E2, E5, E6, E7, E9, E14), pero también porque en Aragón fueron los primeros servicios en ser mancomunados y compartidos por diversos ayuntamientos y de alguna manera se puede considerar que los servicios sociales de atención primaria han sido un germen y catalizador de la división comarcal aragonesa.

No obstante, esa misma división comarcal produce también una enconada crítica al sistema de servicios sociales de atención primaria actual. En primer lugar, porque la división y autonomía comarcal han generado desigualdades entre los ciudadanos aragoneses al tener la comarca potestad reglamentaria en su ordenamiento de ayudas de urgencia, servicio de ayuda a domicilio, etc. Y, en segundo lugar, porque en un sector de la profesión se ha puesto en duda si es la Administración Local la más adecuada para prestar los servicios sociales de atención primaria. No se cuestiona que tengan que ser la puerta de entrada y estar lo más cercanos al ciudadano, pegados al territorio, pero hay profesionales que expresan sus dudas: “¿Para trabajar en un ayuntamiento por qué tengo que ser del ayuntamiento? ¿El colegio es del ayuntamiento? ¿El centro de salud es del ayuntamiento? ¿Por qué tengo que depender de la Administración Local? ¿Para estar más cerca del ciudadano? Si yo voy a ir al mismo pueblo. Eso de que la Administración Local es más cercana... Voy a ser cercana yo que voy al pueblo. Pertenecer a una Administración más grande estabilizaría y consolidaría los servicios” (E8). "Pertenecer a una Administración Local provoca aislamiento y desigualdad respecto a otros sistemas. La falta de carrera profesional y de perspectivas de movilidad o retos profesionales... Se dan casos de depresión y de una sensación de estar laboralmente en una cárcel de oro" (E9).

Asimismo, esta dependencia municipal o comarcal hace que las profesionales se sientan más o menos respaldadas por sus administraciones locales en función de la decisión del Consejo Comarcal correspondiente y la labor personalista del presidente o consejero comarcal, tal y como comenta una compañera: "Se les llena la boca [a los políticos] de servicios sociales, pero a la hora de la verdad les cuesta creérselos" (E3).

A la hora de pensar el futuro de los servicios sociales de atención primaria, reconocen todas las entrevistadas dos riesgos y una necesidad: riesgo de un indeseable aumento de burocratización; riesgo de pérdida de trabajo comunitario; y necesidad de aumento de plantillas de equipos multidisciplinares que además contribuiría a la rebaja de las ratios por profesional. No obstante, ninguna se atreve a vislumbrar cambios de calado en un futuro a corto $y$ medio plazo.

Aunque hay unanimidad al reconocer que en estos últimos años, gracias a la Ley de Dependencia y a la crisis económica, se ha avanzado mucho en la percepción de universalidad y utilidad de los servicios sociales. Son muchos los usuarios que nunca pensaron que tendrían que recurrir a estos servicios y prestaciones, pero persiste también la idea de que, pese al reconocimiento de los servicios sociales, todavía no están equiparados al sistema de salud o al de educación.

\section{Tensión y bipolaridad del sistema de servicios sociales de atención primaria}

Los servicios sociales de atención primaria, desde su inicio y también con la Ley 5/2009 de Servicios Sociales en Aragón, han estado siempre marcados por cierta bipolaridad y tensión. Por un lado, legislativamente hablando, han estado siempre asentados en la Administración municipalista respaldada por la Ley de Bases de Régimen Local, Ley de Administración Local Aragonesa, mancomunidades, proceso comarcalizador, etc. Pero, por otro lado, la planificación estratégica de esta política social, titularidad y ordenación de prestaciones corresponde al Gobierno de Aragón. 
En este contexto, el Decreto 184/2016, que regula los centros de servicios sociales, ha fijado la estructura, personal mínimo, funciones, objetivos, etc., pero luego la titularidad de los centros es de la Administración Local.

Esta bipolaridad produce en Aragón que, tras la supervisión y planificación estratégica del Gobierno de Aragón exista un total de 41 Administraciones Locales diferentes que tienen sus competencias en servicios sociales de atención primaria: los cuatro ayuntamientos de más de $\mathbf{2 0 . 0 0 0}$ habitantes (las tres capitales aragonesas más (alatayud), las treinta y dos comarcas legalmente establecidas y el resto de municipios que pertenecen a la delimitación comarcal de Zaragoza, que al no estar todavía constituida, se agrupan en cinco entidades locales diferentes. Esto significa, por ejemplo, 41 reglamentos de ayudas de urgencia diferentes 041 servicios sociales de ayuda a domicilio diferentes, con sus 41 formularios correspondientes y sus 41 ordenanzas fiscales diferentes, lo que provoca desigualdades entre los ciudadanos aragoneses. De la misma manera, existen 41 convenios colectivos diferentes, con lo cual las condiciones de trabajo de las profesionales del trabajo social también son diferentes pese a ejercer funciones semejantes en cada Administración Local. La homogeneización de criterios, reglamentos, prestaciones etc. es una reivindicación unánime planteada por las profesionales entrevistadas.

Los perjuicios de esta tensión se han manifestado de manera constante a lo largo de estos treinta y cinco años de historia. El inicio de cada servicio fue colocar a una profesional que recibía instrucciones de la dirección provincial de la consejería autonómica competente en cada legislatura, pero que dependía orgánicamente del ayuntamiento o mancomunidad de turno, con lo que el recuerdo de soledad e incomprensión ha surgido en varias entrevistas: "Los inicios fueron muy duros y hubo muy poca ayuda de los servicios provinciales. Te decían 'tú tienes que hacer esto o aquello', pero luego nos dejaban solas ante el peligro. Eso era duro porque te vendían cosas que luego el alcalde te decía: 'de eso ni hablar”' (E12).

La situación no ha mejorado con el tiempo, incluso se puede afirmar que ha empeorado en este sentido con el proceso comarcalizador. Hasta finales de los años noventa, las direcciones provinciales antes indicadas organizaban reuniones periódicas con todos los profesionales de cada provincia para informar y dar las mínimas directrices correspondientes. Tras el proceso de constitución de las comarcas, esas reuniones se han reducido a la mínima expresión, provocando de nuevo sensación de soledad, como puntualiza otra compañera: “¿Nosotras qué hemos ganado como colectivo (profesional) desde que somos comarca? Hemos perdido toda la coordinación. No nos vemos. Antes a todos los profesionales de la provincia nos hacían reuniones mensuales. Ahora ya no" (E6).

\section{Conclusiones}

La creación de una estructura de servicios sociales de atención primaria en España nace del interés y esfuerzo de una serie de profesionales del trabajo social vinculadas, fundamentalmente, a partidos socialistas de finales de los años setenta, y que contó con el apoyo de diversas Administraciones, dirigidas en muchos casos por estos mismos partidos a partir de las victorias en las elecciones tanto generales como municipales y autonómicas en el inicio de los ochenta, y de acuerdo con la anterior aprobación de la Constitución Española de 1978. En Aragón se desarrolló gracias a la dedicación de las primeras profesionales que, a partir de 1983 y con muy poca experiencia, pero con una enorme ilusión y dedicación, se pusieron al frente de la gestión técnica de los recién creados servicios sociales de base.

En un tiempo relativamente corto -menos de tres décadas-, se creó una red de servicios sociales de atención primaria dependiente de la Administración Local (ayuntamientos y comarcas) que cubre el 100\% de la población y está plenamente asentada en el territorio. No obstante, pese a que al sistema de servicios sociales se le reconoce una posición clave para sostener el modelo de Estado de Bienestar, el llamado "cuarto pilar", se constata que en comparación con sanidad y educación no se encuentra al mismo nivel. Casi treinta y cinco años después de la creación del primer servicio social de base rural, ha llegado el momento de reflexionar y evaluar el papel que cumplen en la sociedad, las posibilidades de mejora que tienen y la situación de sus profesionales para conseguir unos servicios de más calidad y efectividad. La actual división competencial y la fragmentación municipalista o "localista” no parecen las formas más indicadas para ofrecer unos servicios adecuados a la ciudadanía del siglo XXI, aun más cuando, desde la aprobación del Catálogo de Servicios Sociales de la Comunidad Autónoma de Aragón (Decreto 143/2011 de 14 de junio) y de acuerdo con la Ley de Dependencia, se regula la prestación de servicios considerados de naturaleza esencial y amparados por derechos subjetivos. Esta situación debería obligar a que la prestación de estos servicios en todos sus aspectos fuese igual para el conjunto de la ciudadanía, independientemente de donde resida o esté censada.

Como resultado preliminar de esta investigación, se dibuja una clara necesidad de mejora en la legislación, en la cartera de servicios, etc., que potencie la equidad y la universalidad (que nadie cuestiona), pero desde una mejor gestión facilitada desde mecanismos y órganos de coordinación que permitan una nueva generación de servicios sociales de atención primaria. 


\section{Referencias bibliográficas}

BÁÑEZ TELLO, T. (2004): El trabajo social en Aragón. El proceso de profesionalización de una actividad feminizada, tesis doctoral, Universidad Rovira i Virgili.

CASADO, D. (1991): Introducción a los Servicios Sociales, Madrid, Editorial Acebo.

Comarcalización de Aragón: compilación en [‘http://www. boa.aragon.es/EBOA/pdf/LibroComarcas.pdf)]; 2007.

CONSTITUCIÓN ESPAÑOLA: Boletín Oficial del Estado; número 311; 29 de diciembre de 1978; pp. 29.313-29.424.

DEPARTAMENTO DE SANIDAD BIENESTAR SOCIAL Y TRABAJO, DIPUTACIÓN GENERAL DE ARAGÓN (1985): LOS Servicios Sociales de Base en Aragón-1984, Zaragoza, Diputación General de Aragón.

FEDAAS (1977): Material de estudio de las III Jornadas Nacionales de Asistentes Sociales, Madrid, Feedas.

GARCÍA HERRERO, G. (1988): "Los Servicios Sociales en el medio rural", Los Servicios Sociales en el medio rural, Madrid, Siglo XXI, pp. 11-106.

- (1988): "Génesis y desarrollo de los Servicios Sociales Comunitarios en el Estado Español”, Servicios Sociales y Política Social, 2. ${ }^{\circ}$ trimestre, número 10, pp. 32-44.

GIL PAREJO, M. (2010): "Los inicios de la construcción del Sistema público de Servicios Sociales desde la perspectiva del Trabajo Social. Unos año prodigiosos, 1978-1988”, Miscelánea Comillas, número 132 , volumen 68 , pp. 185-209.

LAS HERAS, P. y CORTAJARENA, E. (2014): Introducción al Bienestar Social: El libro de las casitas, Consejo General del Trabajo Social, Madrid.

LEY 5/2009 DE SERVICIOS SOCIALES DE ARAGÓN: Boletín Oficial de Aragón, número 132, 10 de julio de 2009, pp. 18.244-18.280.

LEY 6/1982 DE 20 DE MAYO SOBRE SERVICIOS SOCIALES: Boletín Oficial del Estado, número 100, 26 de abril de 2012, pp. 32.117-32.126.

LEY $7 / 1985$ DE 2 DE ABRIL REGULADORA DE LAS BASES DE RÉGIMEN LOCAL, Boletín Oficial del Estado, número 8o, 3 de abril de 1985, pp. 8.945-8.964.

LEY 11/1984 DE 6 DE JUNIO DE SERVICIOS SOCIALES: Boletín Oficial del Estado, 24 de julio de 1984, pp. 21.851-21.854.

MARCO, F. (1988): "Incidencia del Trabajo Social en el desarrollo local. Una experiencia en Aragón”, Servicios Sociales y Política Social, 2.ํㅜ trimestre, número 10, pp. 68-74.

PSOE (1984): Servicios Sociales Municipales, Madrid, Secretaría Federal de Acción Social.

S.S.B. MANCOMUNIDAD ALTO GÁLLEGO (1999): “Una década de Servicios Sociales desde la Mancomunidad Alto Gállego”, Mancomunidad Alto Gállego, (publicación interna). 